\title{
THE ROLE OF SULODEXIDE IN CHRONIC VENOUS INSUFFICIENCY
}

\author{
Rakesh Wahi', Evi Kalodiki², Sanjiv Lakhanpal', Aayushi Rai', Jawed Fareed ${ }^{3}$ \\ 'Center for Vascular Medicine, Greenbelt, MD, USA \\ ${ }^{2}$ Josef Pflug Vascular Laboratory, Ealing Hospital and Imperial College London SW7 2AZ \\ and Thrombosis \& Hemostasis Research Laboratory, Loyola University Medical Centre, \\ Maywood, IL, USA \\ ${ }^{3}$ Thrombosis and Hemostasis Laboratory, Loyola University Medical Centre, Stritch School \\ of Medicine, Maywood, IL, USA
}

\author{
REVIEW PAPER \\ Phlebological Review 2014; 22, 1: 1-5 \\ DOl: $10.5114 /$ pr.2014.46046
}

Submitted: 24.07.2014

Accepted: 1.10.2014

\begin{abstract}
This review paper discusses the mechanism of development of venous pathology and the therapeutic options of sulodexide in venous disease. Chronic venous insufficiency (CVI) is a common pathologic process involving the lower extremities, which presents with diverse clinical findings. The hallmark of the pathologic process is the development of increased venous pressure, which in turn leads to microcirculatory changes. These changes manifest as hypertrophy of the capillaries and endothelial dysfunction. The endothelial dysfunction is accompanied by release of inflammatory mediators as well as a change in the fibrinolytic system, which restricts the skin perfusion. The restricted perfusion, in conjunction with inflammation, is responsible for the development and poor healing of venous ulcers. Sulodexide is a specific glycosaminoglycan (GAG) that consists of $80 \%$ fast moving heparin and $20 \%$ dermatan sulphate and has a unique pharmacological and therapeutic profile. Sulodexide has been shown to reverse the endothelial dysfunction and restore the fibrinolytic balance and is an important adjunct in treating the manifestations of CVI. It also has substantial antithrombotic properties without significantly increasing the risk of haemorrhage. Oral administration of sulodexide is safe and effective since there is no interaction with other drugs. The therapeutic options of sulodexide include CVI, superficial and deep venous thrombosis, and improved healing of venous ulcers.
\end{abstract}

Key words: sulodexide, CVI, SVT, glycosaminoglycans, VV.

\section{ADDRESS FOR CORRESPONDENCE:}

Evi Kalodiki, Josef Pflug Vascular Laboratory, Ealing Hospital and Imperial College London SW7 2AZ and Thrombosis \& Hemostasis Research Laboratory,

Loyola University Medical Centre, Maywood, IL, USA,

e-mail: e.kalodiki@imperial.ac.uk

\section{INTRODUCTION}

Chronic venous insufficiency (CVI) is comprised of a spectrum of symptoms affecting the lower extremities, like pain, oedema, skin changes, and venous ulcers, as a result of venous hypertension arising from the dysfunction of the venous system [1].

Several population studies have found a prevalence rate of CVI ranging from $5-15 \%$ in men and $3-29 \%$ in women $[1,2]$. The Edinburgh vein study found that $9.4 \%$ of men and $6.6 \%$ women suffered from CVI-associated reflux [3]. However, more recently the vein consult program established a worldwide CVI prevalence of $83.6 \%$ [4]. The incidence of CVI increases with age [4]. Other significant risk factors include obesity, pregnancy, history of superficial vein thrombosis, and a family history of varicose veins. Certain behavioural factors such as prolonged standing, sedentary lifestyle, and smoking also increase the risk of developing CVI [1].

In CVI, there is usually a progression of complications, although this progression may not always follow the same sequence. Varicose veins are a common complication associated with CVI, with a female-to-male prevalence ratio of $3: 1$ [1]. Complications of CVI, like venous ulcers, although infrequent, tend to have a poor prognosis with delayed healing time and high rate of recurrence. A recent Cochrane review estimated that up to $1 \%$ of the population in the industrialised world suffer from chronic leg ulcers sometime in their life [5]. It is estimated that about 2.5 million people have CVI in the United States, and of those, about 20\% develop venous ulcers $[1,2]$.

Chronic venous insufficiency has far ranging effects on patient in terms of reduced quality of life, adverse cosmetic implications, and possible disability, as well as a huge financial burden on healthcare systems. It is estimated that 92 out of every 100,000 admissions to hospitals in the United States are due to CVI-related complications [1]. Since CVI has such a high prevalence globally, it is imperative to understand the pathophysiology of CVI development in order to provide effective therapy to at-risk patients.

\section{MECHANISMS OF DEVELOPMENT OF VENOUS PATHOLOGY}

The most common aetiology of CVI is deep vein thrombosis (DVT), which is complicated by post-thrombotic syndrome (PTS) [6]. Both DVT and PTS may 
cause damage to the valves, causing reflux of blood and increased hydrostatic pressure within the veins. Venous pathology develops when the venous pressure increases and drainage of blood is impaired [1]. This may also occur as a result of structural problems such as valvular incompetence of the deep or superficial veins, damage to the perforating veins, venous obstruction, or a combination of these. It may also occur due to functional disturbances such as reduced muscle pump function, which hinders venous return [1]. Rarely the aetiology may be congenital malformation. Valvular incompetence at the junction between superficial and deep veins such as the saphenopopliteal or saphenofemoral junction causes backflow of blood into the superficial veins, leading to tortuous and dilated varicose veins $[1,3]$.

While the effects of venous hypertension on large veins are manifested visibly, there are also adverse effects on the microcirculation associated with CVI, which may not be so apparent. These include elongation, dilatation, tortuosity of the capillary beds, and thickening of the basement membranes with increased collagen and other connective tissue, leading to endothelial damage and widening of inter-endothelial spaces [1]. Furthermore, experimental observations explain the effects on the microcirculation; the trapping of white blood cells and growth factors in the capillaries and postcapillary venules, making these elements unavailable for repair and healing. The adhesion of white blood cells may initiate a cascade of inflammation leading to increased endothelial damage and permeability [1]. There is noted to be an increase in plasma lactoferrin concentration, which may represent local release from the granules of activated neutrophils [7]. Lactoferrin binds to neutrophils, macrophages, natural killer (NK) cells, and fibroblasts controlling the migration, growth, and differentiation of these cells. Local CD11b levels are noted to be increased. The CD11b is an $\alpha$ chain of the leukocyte $\beta_{2}$-integrin Mac- 1 , which mediates binding and extravasation of leukocytes. There is also noted to be a measurable increase in the elastase levels [7].

Concomitant with the increased inflammatory activity, the endothelial dysfunction creates a local imbalance in coagulation and fibrinolytic activity leading to an increased deposition of the Von Willebrand factor (vWF), urokinase-derived plasminogen activator (uPA), as well as plasminogen activator inhibitor-1 (PAI-1) locally. In skin biopsies of the lower extremities of patients with severe CVI, increased deposition of vWF, uPA, and PAI-1 were found in the capillaries [8]. These findings point to a local imbalance in coagulation and fibrinolytic status, which leads to impaired microcirculation and finally to the development of venous leg ulceration.

The dysfunctional endothelium and increased capillary pressure allows for leakage of large protein molecules, which collect and polymerise around the capillaries, resulting in the formation of a cuff around the capillaries
[9]. This is largely a fibrin cuff which contains collagen, laminin, fibronectin, and tenascin. The accumulation of these mediators around the cuff decreases fibrinolysis, leading to deposition of more fibrin and thickening of the cuff. This peri-capillary fibrin cuff restricts oxygen release into the skin. Under resting conditions the mean capillary pressure is higher in patients with CVI as compared to controls. With simulated muscle contraction there is a higher and faster increase in capillary pressure. At the edge of an ulcer, hypoxia is extreme and cutaneous vascular reserve, which is the ability to increase laser major flux after a three-minute arterial occlusion, is exhausted. This lack of vascular reserve prevents the regulation of blood flow to meet instantaneous changes in tissue demand. Therefore, skin blood flow is no longer actively regulated in patients with CVI. These changes are accompanied by an increase in the levels of platelet-derived growth factor (PDGF) as well as by up regulation of vascular endothelial growth factor (VEGF). There is greater expression of VEGF in patients with varicose veins vs. normal controls [10]. These patients also show an increase in the expression of factor VIII-related antigen and adhesion molecules.

One of the adhesion molecules up regulated is intercellular adhesion molecule-1 (ICAM-1) [11]. ICAMs participate specifically in the trafficking of inflammatory cells, in leukocyte effect or functions, in adhesion of antigen-presenting cells to $\mathrm{T}$ lymphocytes, in microbial pathogenesis, and in signal transduction pathways through outside-in signalling events. This adhesion molecule is localised to both the apical and basolateral surface of endothelial cells, making it ideally positioned to facilitate trans-endothelial migration of leukocytes. In fact, ICAM-1 and the vascular cell adhesion molecule-1 (VCAM-1) are considered to represent the most important adhesion molecules for leukocyte recruitment to inflamed sites [10-13].

Increased venous pressure along with microangiopathies also causes fluid and macromolecular metabolic waste products to permeate out of the veins and into the surrounding tissue, triggering an inflammatory reaction. This may commonly manifest as oedema, which may or may not be painful. However, long-standing venous hypertension and inflammation of the surrounding tissue soon results in induration and fibrosis of the subcutaneous tissues, also known as lipodermatosclerosis [1]. Degradation of extracellular matrix component caused by activation of matrix metalloproteinase (MMP) activity is an aetiological factor for lipodermatosclerosis. The overlying skin may develop eczema and pruritus, eventually leading to ulcer formation. Venous ulcers are hard to manage and tend to recur frequently. They may also become infected and cause cellulitis, which can further exacerbate the wound healing process. As a result, patients with venous ulcers require years of specialist wound care and may never achieve complete healing. 


\section{THERAPEUTIC OPTIONS - SULODEXIDE}

Various therapeutic options have been established for CVI, ranging from conservative management with specialist wound care and compression stockings to sclerotherapy and endovenous ablation of varicose veins, open surgical treatment including valvular reconstruction, ligation, and phlebectomies. Newer antithrombotic medications such as sulodexide have been introduced as a therapeutic option $[14,15]$.

Sulodexide is a purified glycosaminoglycan (GAG) used in the treatment of CVI. It is composed of a mixture of $80 \%$ fast-moving heparin, which has an affinity for antithrombin III, and 20\% dermatan sulphate (DS), which has an affinity for heparin cofactor II [16]. Sulodexide confers substantial antithrombotic properties without significantly increasing the risk of haemorrhage. Sulodexide is an orally active drug that undergoes rapid intestinal absorption after administration. This allows the drug to reach high plasma concentrations quickly and permeate into the endothelial layer of the vessel wall. The glycocalyx of the normal endothelial cells contains chondroitin sulphate and dermatan sulphate, which are produced in the endoplasmic reticulum and the Golgi apparatus of the endothelial cells. These proteoglycans contribute significantly to its function, thereby explaining the role of sulodexide.

Sulodexide is metabolised by the liver and excreted by the kidneys. The demographics of patients suffering from CVI may include elderly patients. Therefore, drug interactions with sulodexide are a reasonable concern since many elderly patients suffering from co-morbidities may be under polypharmacy. No drug interferences were found with the concurrent use of sulodexide with diuretics, oral hypoglycaemic drugs, gastric protectors, bronchodilators, tranquilisers, anxiolytics, antibiotics and insulin. Very minor gastrointestinal side effects of sulodexide have been reported, such as transient nausea and dyspepsia [16]. This indicates that oral administration of the drug is safe and effective.

The heparin (HP) fraction of sulodexide has a mean molecular weight of $7 \mathrm{KDa}$ [17]. This fraction has properties similar to LMWH, which allows the drug to achieve a longer half-life, oral bioavailability, as well as decreased pro-haemorrhagic effects $[16,17]$. The DS fraction of sulodexide has a mean molecular weight of $25 \mathrm{kDa}$. Dermatan sulphate has been shown to reduce post-operative DVT in humans [18]. Newer evidence suggests that DS also plays a major role in inhibiting MMP, enzymes that are instrumental in extracellular matrix remodelling, thereby giving sulodexide a protective effect against vessel wall damage and inflammation in CVI [19].

Sulodexide also has extensive thrombolytic and fibrinolytic properties. It has been shown to stabilise glycocalyx and restore depressed endothelial function to near normal levels [20]. The glycocalyx is the site of release of important mediators such as antithrombin III, heparin cofactor II, and tissue factor pathway inhibitor (TFPI). It has been shown to induce tissue plasminogen activator (TPA) and inhibit PAI-1, especially in patients with diabetes $[21,22]$. In a double-bind, placebo-controlled, crossover study in patients with peripheral vascular disease or recurrent venous thrombosis, a marked reduction of PAI- 1 activity $(p<0.001)$ and plasma fibrinogen $(p<0.01)$, as well as increased fibrinolytic activity $(p<0.01)$, were observed after sulodexide was administered orally at $100 \mathrm{mg} /$ day for 30 days [21]. In a study conducted on patients with hyper-viscosity syndrome, the authors reported a remarkable reduction in PAI-1, as well as increased activity of TPA [22].

Sulodexide also exerts its anti-inflammatory effects by inhibiting leukocyte adhesion to vessel walls. It inhibits several cytokines and pro-inflammatory factors like interleukin-6 (IL-6), transforming growth factor $\beta 1$ (TGF- $\beta 1$ ), as well as ICAM-1 [23]. In addition, release of P-selectin, tissue necrosis factor (TNF), and platelet-activating factor $(\mathrm{PAF})$ are also reduced by sulodexide.

In a randomised, double-blind, placebo-controlled study on patients with CVI, sulodexide was shown to significantly reduce the number of complications and symptoms such as pain, oedema, cramps and paraesthesia [24]. The authors also reported a parallel reduction in limb venous pressure. An open-controlled, prospective study conducted in Spain on the safety of sulodexide as a treatment for DVT compared to adjusted dose acenocoumarol concluded that there were no differences in DVT recurrence rates between the two agents, and that sulodexide patients were less prone to haemorrhages [25]. In Italy, a similar study was conducted on 405 patients with documented DVT [26]. These patients, after completing their predefined course of oral anticoagulants, were randomised to receive or not receive oral sulodexide. After 6,12 , and 24 months of sulodexide treatment, the authors reported a significant difference in the incidence of DVT recurrences between the sulodexide and control patients at 24 months. The DVT recurrence rate was $7.4 \%$ in the sulodexide group vs. $17.9 \%$ in the control group. Prophylactic measures to avoid venous thromboembolic complications are particularly important in the elderly population, who may be suffering from mobility disturbances. There has been a study on elderly patients, mean age 71.6 years, with hemiparesis, who were at a high risk of thromboembolic complications and suffering from various co-morbidities [27]. In this study $60 \mathrm{mg}$ of sulodexide was injected intravenously (i.v.) twice daily for one month, followed by oral $50 \mathrm{mg} /$ day of sulodexide for a further month. No evidence of thrombosis was discovered during the two months of treatment in any of the venous, cerebral, or cardiac systems.

Sulodexide was also shown to have benefits for patients affected by venous ulcers in the lower limbs [28, 29]. As previously discussed, venous stasis and hypertension can cause deterioration of endothelial cells and trigger 
inflammation in the microcirculation, eventually leading to ulceration. A randomised, placebo-controlled study was performed on patients affected by venous ulcers of the lower limbs, using sulodexide $60 \mathrm{mg}$ once daily administered intramuscularly (i.m.) for 20 days, followed by $100 \mathrm{mg}$ once daily orally for 70 days. Patients who received sulodexide achieved complete resolution of venous ulcers at both 2 and 3 months of treatment ( $p=$ $=0.018$ and $p=0.004$, respectively). The fibrinogen levels were also significantly decreased.

\section{CONCLUSIONS}

Randomised control trials from different countries have suggested that sulodexide is a safe and effective drug, which has good oral bioavailability and a longer half-life. It exerts a wide range of effects that target all aspects of venous pathology, thrombosis, inflammation, and endothelial damage. It has been shown to be effective in both DVT prophylaxis and in treating venous insufficiency including ulcers, thereby targeting the entire spectrum of CVI. Recently, the American Society for Vascular Surgery and the American Venous Forum have included sulodexide in their clinical guidelines along with compression therapy for people suffering from CVI, especially those exhibiting symptoms of pain and oedema [30].

\section{References}

1. Eberhardt R.T., Raffetto J.D. Chronic venous insufficiency. Circulation 2005; 111: 2398-2409.

2. Brand F.N., Dannenberg A.L., Abbott R.D., Kannel W.B. The epidemiology of varicose veins: the Framingham Study. Am J Prev Med 1988; 4: 96-101.

3. Ruckley C.V., Evans C.J., Allan P.L., Lee A.J., Fowkes F.G. Chronic venous insufficiency: clinical and duplex correlations. The Edinburgh Vein Study of venous disorders in the general population. J Vasc Surg 2002; 36: 520-525.

4. Rabe E., Guex J.J., Puskas A., Scuderi A., Fernandez Quesada F. Epidemiology of chronic venous disorders in geographically diverse populations: results from the Vein Consult Program. Int Angiol 2012; 31: 105-115.

5. O’Meara S., Cullum N., Nelson E.A., Dumville J.C. Compression for venous leg ulcers. Cochrane Database Syst Rev 2012; 11: CD000265.

6. White J.V., Ryjewski C. Chronic venous insufficiency. Perspect Vasc Surg Endovasc Ther 2005; 17: 319-327.

7. Boisseau M.R. Leukocyte involvement in the signs and symptoms of chronic venous disease. Perspectives for therapy. Clin Hemorheol Microcirc 2007; 37: 277-290.

8. Kolbach D.N., Hamulyak K., Prins M.H., Neumann H.A., Cleutjens J.P. Severity of venous insufficiency is related to the density of microvascular deposition of PAI-1, uPA and von Willebrand factor. Vasa 2004; 33: 19-24.

9. Junger M., Klyscz T., Hahn M., Rassner G. Disturbed blood flow regulation in venous leg ulcers. Int J Microcirc Clin Exp 1996; 16: 259-265.

10. Florez A., De Haro J., Bleda S., Varela C., Esparza L., Acin F. Analysis of vascular endothelial growth factor gene expression in the tissues of patients with chronic venous insufficiency. Phlebology 2013; 28: 32-37.

11. Almenar-Queralt A., Duperray A., Miles L.A., Felez J., Altieri D.C. Apical topography and modulation of ICAM-1 expression on activated endothelium. Am J Pathol 1995; 147: 1278-1288.

12. Hubbard A.K., Rothlein R. Intercellular adhesion molecule-1 (ICAM-1) expression and cell signaling cascades. Free Radic Biol Med 2000; 28: 1379-1386.

13. Koning G.A., Schiffelers R.M., Storm G. Endothelial cells at inflammatory sites as target for therapeutic intervention. Endothelium 2002; 9: 161-171.

14. Andreozzi G.M. Sulodexide in the treatment of chronic venous disease. Am J Cardiovasc Drugs 2012; 12: 73-81.

15. Andreozzi G.M. Editorial: sulodexide, an old drug with recent renewed interest. Curr Vasc Pharmacol 2013; 11: 352-353.

16. Lasierra-Cirujeda J., Coronel P., Aza M., Gimeno M. Use of sulodexide in patients with peripheral vascular disease. J Blood Med 2010; 1: 105-115.

17. Coccheri S., Mannello F. Development and use of sulodexide in vascular diseases: implications for treatment. Drug Des Devel Ther 2014; 8: 49-65.

18. Di Carlo V., Agnelli G., Prandoni P., Coccheri S., Gensini G.F., Gianese F., Mannucci P.M. Dermatan sulphate for the prevention of postoperative venous thromboembolism in patients with cancer. DOS (Dermatan sulphate in Oncologic Surgery) Study Group. Thromb Haemost 1999; 82: 30-34.

19. Mannello F., Raffetto J.D. Matrix metalloproteinase activity and glycosaminoglycans in chronic venous disease: the linkage among cell biology, pathology and translational research. Am J Transl Res 2011; 3: 149-158.

20. Broekhuizen L.N., Lemkes B.A., Mooij H.L., Meuwese M.C., Verberne H., Holleman F., Schlingemann R.O., Nieuwdorp M., Stroes E.S., Vink H. Effect of sulodexide on endothelial glycocalyx and vascular permeability in patients with type 2 diabetes mellitus. Diabetologia 2010; 53: 2646-2655.

21. Ceriello A., Quatraro A., Marchi E., Barbanti M., Giugliano D. Impaired fibrinolytic response to increased thrombin activation in type 1 diabetes mellitus: effects of the glycosaminoglycan sulodexide. Diabetes Metab 1993; 19: 225-229.

22. Agrati A.M., Mauro M., Savasta C. A double-blind, cross-over, placebo-controlled study of the profibrinolytic and antithrombotic effects of oral sulodexide. Adv Ther 1992; 9: 147-155.

23. Borawski J., Dubowski M., Pawlak K., Mysliwiec M. Effect of sulodexide on plasma transforming growth factor-betal in healthy volunteers. Clin Appl Thromb Hemost 2010; 16: 60-65.

24. Saviano M., Maleti O., Liguori L. Double-blind, double-dummy, randomized, multi-centre clinical assessment of the efficacy, tolerability and dose-effect relationship of sulodexide in chronic venous insufficiency. Curr Med Res Opin 1993; 13: 96-108.

25. Cirujeda J.L., Granado P.C. A study on the safety, efficacy, and efficiency of sulodexide compared with acenocoumarol in secondary prophylaxis in patients with deep venous thrombosis. Angiology 2006; 57: 53-64.

26. Errichi B.M., Cesarone M.R., Belcaro G., Marinucci R., Ricci A., Ippolito A., Brandolini R., Vinciguerra G., Dugall M., Felicita A., Pellegrini L., Gizzi G., Ruffini M., Acerbi G., Bavera P., Renzo A.D., Corsi M., Scoccianti M., Hosoi M., Lania M. Prevention of recurrent deep venous thrombosis with sulodexide: the SanVal registry. Angiology 2004; 55: 243-249. 
27. Degiglio V., Guida C. Prevention of vascular complications from immobility using suledoxide. Med Praxis 1990; 11: 1-7.

28. Coccheri S., Scondotto G., Agnelli G., Aloisi D., Palazzini E., Zamboni V. Randomised, double blind, multicentre, placebo controlled study of sulodexide in the treatment of venous leg ulcers. Thromb Haemost 2002; 87: 947-952.

29. Andreozzi G.M. Role of sulodexide in the treatment of CVD. Int Angiol 2014; 33: 255-262.

30. Gloviczki P., Comerota A.J., Dalsing M.C., Eklof B.G., Gillespie D.L., Gloviczki M.L., Lohr J.M., McLafferty R.B., Meissner M.H., Murad M.H., Padberg F.T., Pappas P.J., Passman M.A., Raffetto J.D., Vasquez M.A., Wakefield T.W.; Society for Vascular Surgery; American Venous Forum. The care of patients with varicose veins and associated chronic venous diseases: clinical practice guidelines of the Society for Vascular Surgery and the American Venous Forum. J Vasc Surg 2011; 53 (5 Suppl): $2 S-48 S$. 